\title{
Canonical Ensemble Model for the Black Hole Quantum Tunneling Radiation
}

\author{
Jingyi Zhang* \\ Center for Astrophysics, Guangzhou University, 510006, Guangzhou, China
}

(Dated: April 20, 2021)

\begin{abstract}
In this paper, a canonical ensemble model for the black hole quantum tunneling radiation is introduced. With this model the probability distribution function corresponding to the emission shell is calculated. Comparing with this function, the statistical significance of the quantum tunneling radiation spectrum of black holes is investigated. Moreover, by calculating the entropy of the emission shell, a discussion about the mechanism of information flowing out from the black hole is given too.

PACS number(s): 04.70.Dy
\end{abstract}

Keywords: canonical ensemble model, black hole, quantum tunneling, information puzzle

\section{INTRODUCTION}

In 2000, Parikh and Wilczek presented an approach to calculate the emission rate at which particles tunnel across the event horizon [1 3]. The main idea is that if the energy conservation is taken into account, the emission process will be a quantum tunneling, and the barrier will be determined by the self-gravitation interaction of the emission particle. In order to keep the spherical symmetry of the space time during the emission process, Parikh and Wilczek treat the tunneling particle as a spherical shell (or emission shell). In this way a corrected spectrum, which is accurate to a first order approximation, is given. Their result is considered to be in agreement with an underlying unitary theory, and support the information conservation during the emission process of the particles. Following this method, many static or stationary rotating black holes were studied, and similar results were obtained [4 26]. According to the Parikh-Wilczek tunneling framework, for a Schwarzschild black hole the corrected emission spectrum is

$$
\Gamma \sim e^{\Delta S_{B H}}=e^{-8 \pi M \omega\left(1-\frac{\omega}{2 M}\right)},
$$

where $\omega$ is the energy of the emitted particle (emission shell), $M$ is the total mass of the black hole. If $\omega$ is so small that we can ignore the second order term, then we have

$$
\Gamma \sim e^{\Delta S_{B H}} \approx e^{-8 \pi M \omega}=e^{-\beta \omega},
$$

here $\beta=\frac{1}{T}=-8 \pi M$. On the other hand, the spectrum given by Hawking is [27, 28]

$$
N_{\omega}^{2}=\frac{1}{e^{\beta \omega}-1},
$$

where $N_{\omega}^{2}$ denotes the intensity of the positive energy particle flux outside and close to the event horizon, $\omega$ is the energy level of a single emitted particle. In the condition of classical limit, $e^{\beta \omega} \gg 1$, the Hawking radiation spectrum

\footnotetext{
* E-mail: physicz@yahoo.cn
} 
will become as follows

$$
N_{\omega}^{2}=e^{-\beta \omega}
$$

Since $N_{\omega}^{2} \propto \Gamma$, some people think that Eq.(2) and Eq.(4) are the same, and therefore the Parikh-Wilczek's tunneling framework and their tunneling radiation spectrum Eq.(1) give a semi-classical correction to the Hawking radiation spectrum Eq.(3). In fact, Eq.(11) and Eq.(3) are very different. In Eq.(3), $\omega$ is the energy level of a single emitted particle, whereas in Eq.(1) it denotes the energy of an emitted spherical shell. That is, in Parikh-Wilczek's tunneling framework, the $\omega$ is the total energy of a composite particle which contains a number of emitted particles and constructs a spherical shell. Thus, Eq.(11) and Eq.(3) reflect different statistical significance. Moreover, most people think that the greatest success of the Parikh-Wilczek's tunneling framework is the consistency with the conservation of information. However, what is the mechanism of information flowing out the black hole? How can an emission shell carry away information? In this paper, we first attempt to propose a canonical ensemble model to determine the statistical significance of the tunneling radiation spectrum. Then, with this model we calculate the entropy of the emission shell and discuss the mechanism of information flowing out from the hole. We use the Planck units $c=G=\hbar$ throughout the paper.

\section{CANONICAL ENSEMBLE MODEL CORRESPONDING TO THE BLACK HOLE QUANTUM TUNNELING RADIATION}

As described in section I, in Parikh-Wilczek's tunneling framework, the tunneling particle should be a spherical shell (or S-wave) to keep the spherical symmetry of the space time during the emission process. It is actually equivalent to treat a tunneling spherical shell as a composite particle consisting of many particles. In this framework, the tunneling and the shrinking of the black hole take place at the same time. The tunnelling speed of a Spherical shell is 29]

$$
\dot{r}=\frac{1}{2}\left(1-\frac{2(M-\omega)}{r}\right),
$$

Obviously, in the vicinity of the horizon, the speed of the tunneling shell is very slow, infinitely close to zero, and therefore, we can think that it reaches a thermal equilibrium and has the same temperature with the black hole. Without loss of generality, we postulate that this composite particle consists of identical particles, and we can treat this spherical shell as a thermodynamical system. We imagine that the black hole acts as a large heat source, and together with the outgoing spherical shell constitutes an isolated system. Therefore, we can investigate the emission shell with the canonical ensemble theory.

Suppose we have $N$ identical black hole-emission shell systems, which constitute a mixture ensemble. let us define the statistical operator

$$
\hat{\rho}=\sum_{i}\left|\psi_{i}\right\rangle P_{i}\left\langle\psi_{i}\right|
$$

where $\left|\psi_{i}\right\rangle$ denotes the quantum states of the emission shell, $P_{i}$ is the probability of the state $\left|\psi_{i}\right\rangle$.

For a canonical ensemble system, we have

$$
P_{i}=\frac{\Omega_{B H}\left(E-E_{i}\right)}{\Omega(E)}
$$


where $E$ and $E_{i}$ denote the total energy of the isolated system and the energy of the emission shell, respectively. $\Omega_{B H}\left(E-E_{i}\right)$ is the number of microscopic states of the black hole, and $\Omega(E)$ is that of the isolated system. Since $E_{i} \ll E$, in order to obtain the statistical operator $\hat{\rho}$ and compare it with the radiation spectrum of the Parikh-Wilczek tunnelling framework, we expand $\ln \Omega_{B H}\left(E-E_{i}\right)$ into the form of Taylor series. Namely,

$$
\ln \Omega_{B H}\left(E-E_{i}\right)=\ln \Omega_{B H}(E)+\left(\frac{\partial \ln \Omega_{B H}}{\partial E_{B H}}\right)_{E_{B H}=E}\left(-E_{i}\right)+\frac{1}{2}\left(\frac{\partial^{2} \ln \Omega_{B H}}{\partial E_{B H}^{2}}\right)_{E_{B H}=E}\left(-E_{i}\right)^{2}+\cdots
$$

Obviously,

$$
\begin{gathered}
\left(\frac{\partial \ln \Omega_{B H}}{\partial E_{B H}}\right)_{E_{B H}=E}=\beta=\frac{1}{K_{B} T}, \\
\left(\frac{\partial^{2} \ln \Omega_{B H}}{\partial E_{B H}^{2}}\right)_{E_{B H}=E}=\frac{\partial \beta}{\partial E_{B H}}=-\frac{K_{B} \beta^{2}}{C_{B H}},
\end{gathered}
$$

here $C_{B H}$ is the heat capacity of the black hole, $T$ is the Hawking temperature. If we calculate the Eq.(8) to the second order approximation, we obtain

$$
P_{i}=\frac{\Omega_{B H}\left(E-E_{i}\right)}{\Omega(E)}=\frac{1}{Z} e^{-\beta E_{i}-\frac{K_{B}}{2 C_{B H}} \beta^{2} E_{i}^{2}}
$$

where

$$
Z=\sum_{i} e^{-\beta E_{i}-\frac{K_{B}}{2 C_{B H}} \beta^{2} E_{i}^{2}}
$$

Therefore, we have

$$
\begin{aligned}
\hat{\rho} & =\sum_{i}\left|\psi_{i}\right\rangle \frac{1}{Z} e^{-\beta E_{i}-\frac{K_{B}}{2 C_{B H}} \beta^{2} E_{i}^{2}}\left\langle\psi_{i}\right| \\
& =\frac{1}{Z} e^{-\beta \hat{H}-\frac{K_{B}}{2 C_{B H}} \beta^{2} \hat{H}^{2}},
\end{aligned}
$$

where $\hat{H}$ is the Hamiltonian operator of the canonical system. For a Schwarzschild black hole, we have

$$
E=M, \quad T=\frac{1}{8 \pi M K_{B}}, \quad E_{i}=\omega, \quad C_{B H}=-\frac{K_{B} \beta^{2}}{8 \pi} .
$$

Substituting Eq.(15) into Eq.(11) we get

$$
P_{i} \propto e^{-8 \pi M \omega\left(1-\frac{\omega}{2 M}\right)}=e^{\Delta S_{B H}},
$$

and

$$
\hat{\rho}=\frac{1}{Z} e^{-8 \pi M K_{B} \hat{H}+4 \pi \hat{H}^{2}}
$$

That is, we obtain

$$
P_{i}=\Gamma \propto e^{\Delta S_{B H}}
$$

This result tells us that if we treat the black hole together with the emission shell as an isolated system, with the canonical ensemble, we will obtain a probability distribution function $P_{i}$, which is the same as the emission rate of a spherical shell, $\Gamma$. However, in our canonical ensemble model, $\omega$ denotes the total energy of the system, and the $P_{i}$ denotes the probability of the system staying at a macrostate with the total energy $\omega$. Of course, if we consider the emission shell as an identical particles system, at the first order accuracy, we can obtain the same formula as that of Eq.(41). Moreover, since this emission shell contains lots of microstates, as it is emitted from the hole, it surely carrys away a lot of information. That is, there is an out-going information flux near the horizon during the emission process. Now, the most interesting question is: Is the total information of the black hole-emission shell system conservative? 


\section{ENTROPY AND INFORMATION OF THE EMISSION SHELL}

In order to discuss the information puzzle during the emission process, we first calculate the entropy of the emission shell. Let us define a entropy operator

$$
\hat{S}=-K_{B} \ln \hat{\rho}
$$

Then, the mean value of the entropy is

$$
S \equiv\langle\hat{S}\rangle=\operatorname{tr}(\hat{\rho} \hat{S})=-K_{B} \operatorname{tr}(\hat{\rho} \ln \hat{\rho})
$$

The exact computation about the entropy is very difficult. An alternative approach is treating the emission shell as an identical particles system and calculating its entropy with the modified brick-wall method-the thin film model [30, 31]. That is, we treat the emission shell as a thin film staying near the hole.

Let us take the Schwarzschild black hole as an example, and review the thin film model of the entropy computation. The line element of the Schwarzschild black is

$$
d s^{2}=-\left(1-\frac{2 M}{r}\right) d t^{2}+\left(1-\frac{2 M}{r}\right)^{-1} d r^{2}+r^{2} d \Omega^{2} .
$$

The entropy of a thin film composed of identical particles near the hole can be expressed as an integral 31$]$

$$
S=\frac{8 \pi^{3}}{45 \beta^{3}} \int_{r_{H}+\epsilon}^{r_{H}+\epsilon+\delta} \frac{r^{2}}{f^{2}} d r
$$

where $r_{H}=2 M$ is the location of the event horizon, and

$$
f=1-\frac{2 M}{r} .
$$

In Eq. (22), $\epsilon$ is a cutoff and $\delta$ is the thickness of the thin film.

Now we calculate the entropy of the emission shell staying outside the event horizon. The thickness of the emission shell approximately equal to $r_{H}(M)-r_{H}(M-\omega)=2 \omega$. Moreover, considering the self-gravitation of emission particle, for a emission shell the effective space time should be $1-3]$

$$
d s^{2}=-\left(1-\frac{2(M-\omega)}{r}\right) d t^{2}+\left(1-\frac{2(M-\omega)}{r}\right)^{-1} d r^{2}+r^{2} d \Omega^{2}
$$

That is, when we calculate the entropy of the emission shell with the Eqs.(22) and (23), we should replace $M$ with $M-\omega$, and the entropy of the emission shell should be written as

$$
S=\frac{8 \pi^{3}}{45 \beta^{3}} \int_{r_{H}(M-\omega)+\epsilon}^{r_{H}(M-\omega)+\epsilon+2 \omega} \frac{r^{4}}{(r-2 M+2 \omega)^{2}} d r
$$

where $r_{H}(M-\omega)$ is the radial coordinate of the event horizon, $\epsilon$ is the coordinate distance between the emission shell and the horizon. By using the theorem of mean value we obtain

$$
S=\frac{8 \pi^{3}}{45 \beta^{3}} \frac{r_{\xi}^{4}}{(\omega+\epsilon)^{2}} 2 \omega
$$

where

$$
r_{H}(M-\omega)+\epsilon<r_{\xi}<r_{H}(M-\omega)+\epsilon+2 \omega
$$


In general, $\epsilon \gg \delta=2 \omega, r_{\xi} \approx 2 M$. Substituting $\beta=\frac{1}{T}=8 \pi M$ and $r_{\xi} \approx 2 M$ into Eq.(26), we have

$$
S \approx \frac{1}{720 \pi \epsilon^{2}} 8 \pi M \omega\left(1-\frac{\omega}{2 M}+\frac{\epsilon}{2 M}\right) .
$$

In the following, We discuss Eq.(28) for three cases:

1) If we let $\epsilon=\frac{1}{\sqrt{720 \pi}}$, then we obtain

$$
\begin{aligned}
S & \approx 8 \pi M \omega\left(1-\frac{\omega}{2 M}\right)+\sqrt{\frac{\pi}{45}} \omega \\
& =\Delta S_{B H}+\sqrt{\frac{\pi}{45}} \omega .
\end{aligned}
$$

The total entropy of the black hole-emission shell system is

$$
S_{\text {total }}=S_{B H}+S=\frac{A_{H}(M)}{4}+\sqrt{\frac{\pi}{45}} \omega \neq \frac{A_{H}(M)}{4} .
$$

It means that the total entropy of the black hole-emission shell system is greater than that of the black hole before emitting the shell. In fact, according to the definition of information 32 ]

$$
I=S_{\max }-S_{\text {total }}
$$

the information $I$ decrease after emitting a shell. It means that information lose during the emission of the black hole.

2) If $\epsilon<\frac{1}{\sqrt{720 \pi}}$, then we have

$$
\begin{gathered}
S>\Delta S_{B H}+\sqrt{\frac{\pi}{45}} \omega \\
S_{\text {total }}=S_{B H}+S>\frac{A_{H}(M)}{4}+\sqrt{\frac{\pi}{45}} \omega \neq \frac{A_{H}(M)}{4} .
\end{gathered}
$$

It also means that the information $I$ decrease.

3) If $\epsilon>\frac{1}{\sqrt{720 \pi}}$, the shell is far away from the event horizon. In this case, the velocity of the shell can not be ignored, and the thermal equilibrium with the horizon does not exist, and therefore, the above method of calculating the entropy will no longer adapt.

\section{CONCLUSION}

We have introduced a canonical ensemble model corresponding to the Parikh-Wilczek's tunnelling framework. With this model, we can discuss not only the statistical significance of the quantum tunneling radiation spectrum but also the mechanism of information flowing out from the hole. We showed that the probability distribution function for the canonical ensemble model is the same as the tunnelling rate of the emission particles. It means that the quantum tunnelling rate is, in fact, equal to a probability that the black hole transits from one quantum state corresponding to the mass $M$ to another state corresponding to the mass $M-\omega$. We found that the emission shell contains information and when it is emitted out from the hole, there are information flowing out. However, according to the canonical ensemble model, the total information is not conservative. That is, information lose in the process of emission. It should be mentioned that our canonical ensemble model is different from the model presented in Ref.[33]. In Ref. 33] the authors treated the black hole as a canonical ensemble composed of a naked black hole and the two-dimensional thermodynamic surface (horizon of the black hole). By using the quantum statistical method, they also derived the energy spectrum of the black hole Hawking radiation. 


\section{Acknowledgments}

This research is supported by the National Natural Science Foundation of China (Grant Nos. 11273009, 10873003, 10573005, 10633010) and the National Basic Research Program of China (Grant No. 2007CB815405).

[1] M. K. Parikh, F. Wilczek, Phys. Rev. Lett., 85, 5042(2000) [arxiv: hep-th/9907001].

[2] M. K. Parikh, Int. J. Mod. Phys. D 13,2355(2004) [arXiv: hep-th/0405160.

[3] M. K. Parikh, arXiv: hep-th/0402166,

[4] S. Hemming, E. Keski-Vakkuri, Phys. Rev.D64, 044006(2001).

[5] A. J. M. Medved, Phys. Rev.D66, 124009(2002).

[6] M. A.ves, Int. J. Mod. Phys. D 10, 575(2001).

[7] J. Zhang, Z. Zhao, Mod. Phys. Lett. A 20, 1673(2005).

[8] J. Zhang, Z. Zhao, Phys. Lett. B 618, 14(2005).

[9] W. B. Liu, Phys. Lett. B 634,541(2006).

[10] S. Q. Wu, Q. Q. Jiang, J. High Energy Phys. (3)(2006)Art. No.079.

[11] J. Zhang, Z. Zhao, Nucl. Phys. B 725, 173(2005).

[12] J. Zhang, Z. Zhao, J. High Energy Phys. (10)(2005)Art. No.055.

[13] J. Zhang, Z. Zhao, Phys. Lett. B 638, 110(2006).

[14] J. Zhang, Z. Zhao, Acta Phys. Sin.(in chinese) 55, 3796(2006).

[15] J. Zhang, Z. Zhao, Mod. Phys. Lett. A 21, 1865(2006).

[16] J. Zhang, Jun-Hui Fan, Chinese Physics 16, 3879(2007).

[17] J. Zhang, Jun-Hui Fan, Phys. Lett. B 648, 133(2007).

[18] J. Zhang, Mod. Phys. Lett. A 22, 1821(2007).

[19] J. Zhang, Phys. Lett. B 668, 353(2008).

[20] J. Zhang, Phys. Lett. B 675, 14(2009).

[21] J. Zhang, Z. Zhao, Sci. China-phys. Mech. Astron. 53, 1427(2010).

[22] Q. Q. Jiang, S. Q. Wu, and X. Cai, Phys. Rev. D73, 064003(2006).

[23] R. Banerjee, B. R. Majhi, Phys. Lett. B 662, 62(2008).

[24] R. Banerjee, B. R. Majhi, S. Samanta, arXiv: 0801.3583.

[25] R. Banerjee, B. R. Majhi, arXiv: 0805.2220.

[26] S. Kar, Phys. Rev. D 74, 126002(2006)[arxiv: hep-th/0607029].

[27] S. W. Hawking, Nature (London) 248, 30(1974).

[28] S. W. Hawking, Commun. Math. Phys.43, 199(1975).

[29] J. Zhang, Z. Zhao, Phys. Rev.D 83, 064028(2011).

[30] G. t' Hooft, Nucl. Phys. B 256, 727(1985).

[31] X. Li, Z. Zhao, Phys. Rev.D 62, 104001(2000).

[32] D. N. Page, hep-th/9305040v5.

[33] R. Zhao, Li-Chun Zhang, Shuang-Qi Hu, Acta Phys. Sin.(in chinese) 55, 3898(2006). 